\title{
Phylogenomics of 'Discosea': A new molecular phylogenetic perspective on Amoebozoa with flat body forms
}

\author{
Yonas I. Tekle ${ }^{\mathrm{a},{ }^{*}}$, O. Roger Anderson ${ }^{\mathrm{b}}$, Laura A. Katz ${ }^{\mathrm{c}, \mathrm{d}}$, Xyrus X. Maurer-Alcalác,d, \\ Mario Alberto Cerón Romero ${ }^{\mathrm{c}, \mathrm{d}}$, Robert Molestina ${ }^{\mathrm{e}}$, \\ ${ }^{a}$ Spelman College, 350 Spelman Lane Southwest, Atlanta, GA 30314, USA, \\ ${ }^{b}$ Biology, Lamont-Doherty Earth Observatory of Columbia University, Palisades, New \\ York, USA \\ ${ }^{c}$ Department of Biological Sciences, Smith College, Northampton, Massachusetts, USA, \\ ${ }^{d}$ Graduate Program in Organismic and Evolutionary Biology, University of \\ Massachusetts, Amherst, Massachusetts, USA, \\ ${ }^{e}$ American Type Culture Collection, Manassas, Virginia, USA,
}

*Corresponding Author: Yonas I. Tekle, Spelman College, 350 Spelman Lane Southwest, Atlanta, GA 30314---Telephone number: 404-270-5779; e-mail: ytekle@ spelman.edu 


\begin{abstract}
The majority of amoeboid lineages with flattened body forms are placed under a taxonomic hypothetical class 'Discosea' sensu Smirnov et al. (2011), which encompasses some of the most diverse morphs within Amoebozoa. However, its taxonomy and phylogeny is poorly understood. This is partly due to lack of support in studies that are based on limited gene sampling. In this study we use a phylogenomic approach including newly-generate RNA-Seq data and comprehensive taxon sampling to resolve the phylogeny of 'Discosea'. Our analysis included representatives from all orders of 'Discosea' and up to 550 genes, the largest gene sampling in Amoebozoa to date. We conducted extensive analyses to assess the robustness of our resulting phylogenies to effects of missing data and outgroup choice using probabilistic methods. All of our analyses, which explore the impact of varying amounts of missing data, consistently recover well-resolved and supported groups of Amoebozoa. Our results neither support the monophyly nor dichotomy of 'Discosea' as defined by Smirnov et al. (2011). Rather, we recover a robust well-resolved clade referred to as Eudiscosea encompassing the majority of discosean orders (seven of the nine studied here), while the Dactylopodida, Thecamoebida and Himatismenida, previously included in 'Discosea,' are nonmonophyletic. We also recover novel relationships within the Eudiscosea that are largely congruent with morphology. Our analyses enabled us to place some incertae sedis lineages and previously unstable lineages such as Vermistella, Mayorella, Gocevia, and Stereomyxa. We recommend some phylogeny-based taxonomic amendments highlighting the new findings of this study and discuss the evolution of the group based on our current understanding.
\end{abstract}

Key Words: RNA-Seq; Transcriptome; Amoeba; Lobosea; Flabellinea; Tubulinea 


\section{Introduction}

The taxonomy and phylogeny of amoeboid lineages currently classified within the eukaryotic clade Amoebozoa have been challenging to study (Adl et al., 2012; Amaral Zettler et al., 2000; Cavalier-Smith et al., 2004; Lahr et al., 2011a; Smirnov et al., 2005; Smirnov et al., 2011; Tekle et al., 2008). While one of the main hurdles is scarcity of diagnosable morphological characters in this group (Lee et al., 1985; Page, 1987;

Rogerson and Patterson, 2002; Schaeffer, 1926), other challenges are the under sampling of taxa, and particularly insufficient gene sampling in molecular phylogenetic studies (Amaral Zettler et al., 2000; Lahr et al., 2011a; Tekle et al., 2008). While making significant advances, studies based on a single or few molecular gene sequences resolve mainly shallow rather than deep nodes (Lahr et al., 2011a; Tekle et al., 2008). Moreover, they support only some of the well-established relationships based on morphology (Lahr et al., 2011a; Smirnov et al., 2011).

Molecular phylogenetic studies in Amoebozoa have been steadily growing albeit with limited gene sampling (Lahr et al., 2011a; Lahr et al., 2013; Tekle et al., 2008). These studies have contributed to our knowledge of the taxonomic breadth of the Amoebozoa, which is far more diverse than originally conceived. Amoebozoa now includes reticulate/filose and flagellate amoeboid forms (Berney et al., 2015; Lahr et al., 2012; Nikolaev et al., 2006; Tekle et al., 2008), in addition to the Mycetozoa (i.e. slime molds) and diverse naked and testate lobose amoebae. Despite these major advances, the monophyly and deep relationships of most of the amoebozoan subclades, hypothesized largely from morphological data, remain unresolved (Kudryavtsev et al., 2014; Lahr et al., 2011a; Tekle et al., 2008). Some confounding factors contributing to the failure of the commonly used genes (e.g. SSU-rDNA and actin) in reconstructing phylogenies in amoebozoans is related to long-branch attraction (Felsenstein, 1978) and complicated gene history (Lahr et al., 2011b). The SSU-rDNA genes of some amoebozoans are amongst the fastest evolving in eukaryotic lineages (Tekle et al., 2008), which is an impediment to phylogenetic reconstruction methods (Lartillot et al., 2007; Pisani, 2004). Similarly, presence of multiple copies of actin (i.e. paralogs) in amoebozoans hinders accurate species tree inferences (Lahr et al., 2011b). 
Resolving deep relationships in Amoebozoa requires increased gene sampling in an effort to amass phylogenetic signal over noise, which might help circumvent some of the above confounding factors. Large scale data from Amoebozoa such as whole genome, transcriptome and expressed sequence tag (EST) are sorely lacking, and limited only to a few lineages (Eichinger et al., 2005; Grant and Katz, 2014; Stanley, 2005); although this will very likely change in the foreseeable future as EST projects focusing on diverse taxonomic groups are steadily emerging (Cavalier-Smith et al., 2015; Grant and Katz, 2014). A recent multigene study of amoebozoans using transcriptome data, with limited taxonomic sampling, showed promise in resolving deep relationships within the group (Cavalier-Smith et al., 2015). In the study reported here, we used a large-scale analysis approach to resolve the phylogeny of one of the most problematic Amoebozoa subclades ('Discosea') encompassing the majority of flat amoebae.

Lobose amoebae are grouped into two large clusters: 'Discosea' and Tubulinea, primarily based on morphology plus limited molecular data (Smirnov et al., 2011). While the Tubulinea has consistently received some support in molecular phylogenetic analyses (Lahr et al., 2011a; Lahr et al., 2013; Tekle et al., 2008), the 'Discosea' as it is currently defined is not recovered in molecular studies (hence we place it in quotes to indicate uncertainty). 'Discosea' was originally erected based on limited molecular data sampling and loosely defined morphological data (Cavalier-Smith et al., 2004). Even though subsequent studies with increased taxon sampling and refined morphological data have improved our understanding of the 'Discosea' (Kudryavtsev et al., 2014; Lahr et al., 2011a; Nikolaev et al., 2006; Smirnov et al., 2005; Tekle et al., 2008), it remains one of the most contentious groups within the Amoebozoa (Smirnov et al., 2011). 'Discosea' sensu Smirnov et al. (2011) is broadly defined as diverse groups of flattened naked amoebae with polyaxial cytoplasmic flow. The group encompasses nine morphotypes of lobose amoebae, compared to three that are found within Tubulinea (Smirnov et al., 2011). As the result of this huge diversity, 'Discosea' is defined largely by the absence of characters that are used to distinguish it from the Tubulinea, rather than by a unifying 
morphological synapomorphy. Thus, it is unclear whether 'Discosea' is a natural clade, or a paraphyletic collection of lineages.

'Discosea' has historically been revised repeatedly and its members redefined, as evidence has emerged from analyses with different taxon sampling and reevaluation of morphological characters (Cavalier-Smith et al., 2004; Smirnov et al., 2005; Smirnov et al., 2011). Smirnov et al. (2011) recognized two discosean subclades, Longamoebia and Flabellinia, based on morphology. Longamoebia includes flat amoebae with pointed subpseudopodia and elongated cell shape, while members of Flabellinia are discoid to fan-shaped without pointed subpseudopodia (Smirnov et al. 2011). Moreover, some members of Longamoebia possess centrosomes, while this feature is not reported in Flabellinia (Smirnov et al. 2011). The dichotomy of 'Discosea' into these two subclades, Longamoebia and Flabellinia, is rarely recovered in molecular analyses involving SSUrDNA or actin (Lahr et al., 2011a; Tekle et al., 2008). Similarly, the phylogenetic affinity of several well-characterized taxa within the discosean subclades is inconsistent (Kudryavtsev et al., 2014; Lahr et al., 2011a; Tekle et al., 2008). 'Discosea' also has the highest number of incertae sedis (lineages of unknown taxonomic placement), 14 of 24, in one recent taxonomy of Amoebozoa (Adl et al., 2012). This list does not include several putative members of 'Discosea' that never or rarely form a group, including members of Dermamoebida (e.g. Mayorella, Dermamoeba), Vannellida (e.g. Pessonella), Himatismenida (e.g. Cochliopodium, Parvamoeba, Gocevia), Trichosida (e.g. Trichosphaerium), and Stygamoebida (e.g. Vermistella, Stygamoeba) (Berney et al., 2015; Kudryavtsev et al., 2014; Lahr et al., 2011a; Tekle et al., 2008).

In this study we seek to resolve the phylogeny of the 'Discosea' including its monophyly, dichotomy into Longamoebia and Flabellinia, and placement of enigmatic taxa using large-scale analysis. We analyze a total of 40 amoebozoans, including 24 putative discoseans, from newly characterized RNA-Seq data and existing EST and genomic data using probabilistic methods. Our findings provide new and deeper insights into the taxonomic composition and evolution of the 'Discosea'. We also report evidence for several novel relationships and find a taxonomic home for some putative discosean 
incertae sedis. Finally, we make taxonomic amendments based on our current understanding of the group.

\section{Materials and Methods}

\subsection{Cultures}

A list of the six amoebae strains characterized for this study, including culture conditions and description of bacteria used as food sources during cultivation, are provided (Table S1). Clydonella sp. ATCC ${ }^{\circledR} 50884^{\mathrm{TM}}$, Gocevia fonbrunei $\mathrm{ATCC}^{\circledR} 50196^{\mathrm{TM}}$, Vermistella antarctica $\mathrm{ATCC}^{\circledR}$ PRA-216 ${ }^{\mathrm{TM}}$, and Parvamoeba monoura $\mathrm{ATCC}^{\circledR}$ PRA- $35^{\mathrm{TM}}$ were grown in a minimum of eight $75 \mathrm{~cm}^{2}$ plastic culture flasks to obtain enough starting material for RNA isolation. Thecamoeba quadrilineata $\mathrm{ATCC}^{\circledR} \mathrm{PRA}-259^{\mathrm{TM}}$ and Unda schaefferi $\mathrm{ATCC}^{\circledR} 50810^{\mathrm{TM}}$ were grown in a minimum of four $10 \mathrm{~cm}$ diameter agar plates. Cultures were incubated for 4-8 days. Adherent amoebae were removed from plastic culture flasks or agar plates and centrifuged at $300 \mathrm{x} \mathrm{g}$. Pellets were resuspended in $600 \mu$ of buffer RLT (Qiagen ${ }^{\circledR}$ ) and frozen at $-80^{\circ} \mathrm{C}$. The minimum cell counts used in RNA extraction were $\geq 5 \times 10^{6}$ cells for ATCC ${ }^{\circledR}$ PRA-259 ${ }^{\mathrm{TM}}$, ATCC $^{\circledR}$ PRA-216 ${ }^{\mathrm{TM}}$, and ATCC $^{\circledR} 50196^{\mathrm{TM}}$, and $\geq 2 \times 10^{7}$ cells for ATCC ${ }^{\circledR}$ PRA- $^{\circ} 5^{\mathrm{TM}^{\mathrm{M}}}, \mathrm{ATCC}^{\circledR} 50884^{\mathrm{TM}}$, and $\mathrm{ATCC}^{\circledR} 50810^{\mathrm{TM}}$.

\subsection{RNA isolation}

Total RNA from lysates above was isolated using the Qiagen ${ }^{\circledR}$ RNeasy ${ }^{\circledR}$ Mini Kit (QIAGEN group, USA) according to the manufacturer's instructions. The concentration of the RNA samples was measured using a Qubit ${ }^{\circledR}$ RNA HS Assay Kit and Qubit ${ }^{\circledR} 2.0$ fluorometer (Life Technologies, Carlsbad, CA). Integrity of RNA was evaluated using the Agilent RNA 6000 Pico Assay kit and an Agilent 2100 Bioanalyzer (Agilent Technologies, USA). Electropherograms obtained from the Agilent 2100 Bioanalyzer were inspected visually. RNA concentration, 28S:18S ratio, and RNA integrity number (RIN) were obtained with the Agilent 2100 Expert Software. 


\subsection{Preparation of libraries and sequencing}

A total RNA input of between 0.5 and $4 \mu \mathrm{g}$ was used in library preparation. Libraries were prepared using the TruSeq Stranded mRNA Library Prep kit (Illumina, San Diego, CA). This kit includes a poly(A) + RNA selection step using oligo (dT) magnetic beads, fragmentation of RNA, followed by first- and second-round cDNA synthesis. Thereafter, the libraries were prepared according to the manufacturer's protocol without further modifications. Library profiles were assessed using the DNA High Sensitivity LabChip kit on an Agilent Bioanalyzer. Libraries were quantified by qPCR using the KAPA Library Quantification Kit for Illumina Libraries (KapaBiosystems, Wilmington, MA). Libraries were diluted to a final concentration of 6-10 pM and run on the Illumina MiSeq, using V2 2x250 cycle flow cells in a paired-end mode. After sequencing, an in-house quality control process was applied to reads that passed the Illumina quality filters (raw reads). The sequences of the Illumina adapters and primers used during the library construction were removed from the whole reads. Low-quality nucleotides (quality value $<20)$ were removed from both ends. The longest sequence without adapters and lowquality bases were maintained. Sequences between the second unknown nucleotide $(\mathrm{N})$ and the end of the read were also trimmed.

\subsection{Sequence Assembly and phylogenetic analysis using bioinformatics pipeline}

We used a variety of approaches to assemble and analyze a total of 5-35M reads from the transcriptomes of each taxon (Table 1). Each taxon is sequenced to at least 30x coverage. Low quality reads, those with a score below 20 , and Illumina adapters sequences were removed using FastQC (http://www.bioinformatics.babraham.ac.uk/projects/fastqc/) and Trimmomatic (version 0.30 SLIDINGWINDOWN:4:15 MINLEN:101; (Bolger et al., 2014), respectively. The remaining transcriptome reads were assembled de novo with SeqMan NGEN (Version 12.2) using default parameters with the only exceptions being that we removed contigs that were $\leq 200 \mathrm{bp}$ in length and had lower than average $5 \mathrm{x}$ coverage. Using custom python scripts, remaining contigs were filtered against a database of RefSeq bacterial and archaeal sequences to enrich the dataset for eukaryotic protein coding sequences. 
We used a taxon-rich phylogenomic pipeline that was developed by Grant and Katz (2014). This pipeline includes 13,104 'orthologous groups' (OGs, essentially clusters of homologs organized in OrthoMCL (http://www.orthomcl.org/orthomcl/) that are used to capture homologs from addition taxa. Because of our interest in placing six newly characterized lineages of Amoebozoa onto phylogeny, we modified the pipeline to include all Amoebozoa plus other eukaryotic lineages with completed genome or transcriptome data (see Table S2). We added the transcriptomes of $G$. fonbrunei, $P$. monoura, V. antarctica, T. quadrilineata, Clydonella sp. and U. schaefferi obtained from this study to the pipeline by blasting the contigs from the transcriptome against the total 13,104 OGs. Using this approach, we identified 550 OGs for phylogenetic analyses because these OGs had 7 or more of our target taxa (i.e. amoebozoans) represented in the final data matrices. All selected OGs of each amoebozoan ingroup taxon were inspected for possible contamination and paralogy issues using single gene phylogenetic analysis before concatenation. The 550 OGs yielded multigene alignments and phylogenies as described below.

We reconstructed phylogenies using probabilistic methods including RAxML (Stamatakis et al., 2008; Stamatakis et al., 2005) and MrBayes v3.2.2 (Ronquist and Huelsenbeck, 2003) as implemented at CIPRES portal (Miller et al., 2010). We conducted extensive analysis on three datasets, full (550 genes, 199347 aa,), 25\%-mask (496 genes, $149123 \mathrm{aa}$ ), and 50\%-mask (400 genes, 97059 aa), that differed in the amount of missing data (see Table 2). We also conducted additional analysis to see the effect of selected evolutionary models and outgroup choice (opisthokonts only, data not shown, vs. major eukaryotic representatives; Fig. 1) on the resulting phylogenies, and found no substantial differences in resulting topologies.

We used ProtTest to determine the best fitting model for each gene (Darriba et al., 2011). Over 95\% (522 out of 550) of the genes analyzed indicated that the LG model was the best fitting model and the remaining had JTT (8 genes), WAG (15 genes), Dayhoff (4 genes) and RtREV (1 gene). For all the latter genes LG was among the top best fitting 
models. Since estimates from ProtTest were similar for $+\mathrm{G}$ and $+\mathrm{G}+\mathrm{I}$, and due to the computational cost of partitioning these data, all of our final RAxML analyses were ran under the PROTGAMMALG model using default parameters as implemented in CIPRES (Berger et al., 2011; Stamatakis et al., 2005). Bootstrap was simultaneously calculated using the same model with 100 replicates (Stamatakis et al., 2008). MrBayes analysis was conducted using the GTR CAT model, to assess the stability of our results to changing models and algorithm in CIPRES. All of our MrBayes analyses did not converge and timed out due to computation restrictions on the CIPRES portal. Although all of our first MrBayes runs timed out (168 hrs) without convergence, we had a near convergence in our second re-run ( $\leq 3$ million iterations) with one dataset (50\%-mask), which generated a similar topology to the RAxML analysis (data not shown). Our final alignment and tree are accessible through TreeBASE (http://purl.org/phylo/treebase/phylows/study/TB2:S18520?x-access-

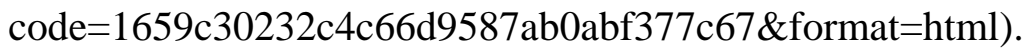

\subsection{Hypothesis testing}

We used Approximately Unbiased (AU) test (Shimodaira, 2002) in order to assess the robustness of newly inferred phylogeny and whether non-recovered hypotheses could be rejected given the data analyzed. A total of seven alternative hypotheses were generated using MacClade (Maddison and Maddison, 2005). These trees were compared to the newly recovered best RAxML tree (Fig. 1) to calculate per-site likelihoods. CONSEL (Shimodaira and Hasegawa, 2001) was used to calculate p-values, from the generated per-site likelihoods, with standard parameters.

\section{Results}

\subsection{Amoebozoa Phylogeny}

The final concatenated data matrices that we analyzed included 40 diverse Amoebozoa and nine outgroup taxa representing major eukaryotic lineages. In all three datasets analyzed (varying in number of characters and genes; Table 2) the monophyly of Amoebozoa was consistently recovered with full support (Fig. 1, Table 2). The overall branching order and support for well-established shallow and intermediate clades in our 
phylogenies were congruent with previous publications (Lahr et al., 2011a). Noteworthy relationships include full support for Mycetozoa and Varipodida as well as recovery of Mycetozoa+Varipodida (Semiconosia) and Tubulinea (represented by only two lineages) in most of our analysis, though the latter with bootstrap support (BS) ranging from weak to strong (Fig. 1, Table 2). We did not recover Conosa or a division of Lobosa into Discosea and Tubulinea in any of our analysis, although these results may be due to limited taxonomic sampling in some areas and/or long-branch attraction among members of Archamoebae (Table S2).

\section{2. 'Discosea' Phylogeny}

Our analyses included a total of 24 putative discoseans representing each taxonomic order based on the taxonomic composition of 'Discosea' sensu Smirnov et al. 2011 and Kudryavtsev et al. 2014 for Pellitidae (Table S2). Each taxonomic order is represented by at least one taxon with more than $50 \%$ of the analyzed characters present in all three datasets (see Table S2) analyzed (e.g. >100,000 aa in the full dataset) except for the order Trichosida (23\% in the full dataset; see Tables 2, S2). The well-supported groups in our phylogenies were robust to possible effects due to missing data (Table 2), taxon sampling, and outgroup choice (data not shown). The full (Fig. 1) and 25\%-mask (Table 2, Fig. S1) datasets generated identical topologies with similar BS supports (Fig. 1, Table 2). The topology of the 50\%-mask (Fig. S2) differed from the above two datasets in that the Tubulinea and Semiconosia were not monophyletic (Table 2, Figs 1, S1,2).

The monophyly of 'Discosea' as defined by Smirnov et al. (2011) was not recovered in any analysis performed for this study (Table 2). Similarly, the AU tests reject the monophyly of 'Discosea' (Table 3). Our analyses also do not recover the monophyly of either Flabellinia or Longamoebia (Fig. 1, Table 2). Instead, we recover a novel robust clade including three orders of Flabellinia (Dactylopodida, Vannellida and Stygamoebida) plus all three orders of Longamoebia (Dermamoebida, Thecamoebida and Centramoebida). This clade, hereafter referred to as Eudiscosea (see below), is consistently recovered in all of our analyses with high BS supports (Fig. 1, Table 2). The taxonomic compositions of the orders comprising the Eudiscosea are recovered as 
expected with few notable exceptions. Mayorella sp., a member of Dermamoebida, is nested within Dactylopodida as a sister group to Vexilliferidae with full support (Table 2). Members of Stygamoebida (Vermistella antarctica) and Himatismenida (Gocevia fonbrunei) group with Thecamoebida and Centramoebida with full support, respectively (Table 1). The clade including Centramoebida and Gocevia also includes Stereomyxa ramosa, a lobose incertae sedis, which groups as sister to Acanthamoeba (Fig. 1, Table 2).

The branching order within the Eudiscosea is well resolved (Fig. 1). Vannellida and Dactylopodida+Mayorella form a sister group relationship with full support in all of our analyses (Figs 1, S1,2, Table 2). This clade is sister to a fully supported clade including Thecamoeba + Vermistella and Stenamoeba (Fig. 1). A clade consisting of Gocevia + (Stereomyxa + Centramoebida) branches basal to the above Eudiscosea members with moderately strong BS support (Table 2). The topology and membership of the analyzed taxa are very robust to taxonomic and gene sampling. Even taxa represented by less than $2 \%$ of the total data (e.g. Acanthamoeba healyi and Vannella simplex) were placed in their respective taxonomic groups with full support (Figs 1, S1,2; Tables 2, S2).

The putative discosean taxa that were never observed to group within the Eudiscosea include Trichosphaerium sp., Pessonella spp., Sapocribrum chincoteaguense and three members of Himatismenida (Cochliopodium minutoidum, Ovalopodium desertum and Parvamoeba monoura; Fig. 1). The three himatismenids form a monophyletic group with full BS support (Fig. 1). The two Pessonella species and S. chincoteaguense also formed a monophyletic group with strong BS support (Table 2). The three himatismenids and Pessonella $+S$. chincoteaguense group as sister taxa consistently in most of our analysis albeit with very weak BS support (Table 2). The placement of Trichosphaerium is variable and inconsistent with any of the proposed taxonomic hypotheses. This taxon was mostly observed to group with the long-branch clade Archamoebae as sister to Tubulinea (Figs 1, S1) and for the 50\%-mask it was found nested within the Mycetozoa and Varipodia clade (Fig. S2). Since the monophyly of 'Discosea' sensu Smirnov et al. (2011) was rejected by our AU tests, we tested membership of these unresolved putative discoseans individually or as a group within the Eudiscosea (see Table 3). Our AU tests 
reject all of the taxonomic combinations, thus further refuting their membership within Eudiscosea clade (Table 3).

\subsection{Placement of newly characterized taxa}

All of the newly sequenced taxa were robustly placed in the resulting phylogenetic tree (Fig. 3). The two vannellid taxa, Clydonella sp. and Unda schaefferi were placed within the Vannellida with full support (Fig. 1). While Parvamoeba monoura grouped with the two members of Himatismenida ( $C$. minutoidum and $O$. desertum) as expected, the putative himatismenid, Gocevia fonbrunei, grouped consistently with a clade consisting of Stereomyxa and Acanthamoeba with full support (Fig. 1). Vermistella antarctica grouped with members of Thecamoebida (S. stenopodia and Thecamoeba quadrilineata) with full support. However, its sister relationship with T. quadrilineata is weakly supported (Figs 1, S1, S2).

\section{Discussion}

\subsection{Amoebozoa Phylogeny}

Our data comprise the largest gene sampling (up to 550 genes, Table 2) and comprehensive taxon sampling (40 lineages) within the Amoebozoa. The results not only confirm well-established relationships within Amoebozoa but also reveal new insights into the evolution of amoebae with flat body shapes. In all of our analyses, Amoebozoa was consistently recovered as a monophyletic group with robust support (Table 2). Moreover, these analyses recovered intermediate to deep relationships including Semiconosia and Tubulinea (Table 2). The latter two relationships had mixed support in our full and 25\%-mask datasets analyses and were not recovered in the 50\%-mask dataset (Table 2). This is due to the nested branching of the two long-branch lineages, Archamoebae and Trichosphaerium (Fig. S2). The placement of these two known problematic lineages in most of our analyses, similar to many ribosomal-based studies (Lahr et al., 2011a; Tekle et al., 2008), was unstable and inconclusive (data not shown). Even though the focus of our study is the 'Discosea', the two deepest Amoebozoan hypothetical assemblages, Conosa and Lobosa, were never recovered in any of our 
analyses (Figs 1, S1,2). A recent multigene study reported the division of Amoebozoa into Conosa and Lobosa (Cavalier-Smith et al., 2015). However, that study was based on much more limited taxon and gene sampling [25]; hence the dichotomy of Amoebozoa will need further scrutiny using taxon rich phylogenomic sampling.

\section{2. 'Discosea' sensu Smirnov et al. (2011) is not monophyletic}

Comprehensive taxonomic and gene sampling provides strong evidence that 'Discosea' is not monophyletic (Table 2), as hypothesized by Smirnov et al. (2011). Our current study also fails to support the division of 'Discosea' into Longamoebia and Flabellinia (Fig. 1, Table 2). This is not surprising since there is neither robust molecular support (Kudryavtsev et al., 2014; Lahr et al., 2011a; Tekle et al., 2008) nor reliable morphological characters that can be used to define these taxonomic hypotheses (Smirnov et al. 2011). As stated above, previous molecular studies that analyzed members of 'Discosea' suffered from limited gene and/or taxon sampling. The 'Discosea' includes lineages of diverse morphology, which creates a challenge to find unifying morphological synapomorphies for 'Discosea' or the two major subgroups in it. Therefore, morphological characters used to define these taxonomic hypotheses are likely homoplasious and need further scrutiny. Similarly, the taxonomic composition of 'Discosea' as defined by Smirnov et al. (2011), differs substantially from our phylogeny (Fig. 1). There are nine putative orders within the 'Discosea', six of which are classified under the subclass Flabellinia and the remaining three in the subclass Longamoebia (Smirnov et al., 2011). The majority of the discosean orders analyzed (seven of nine) form a well-supported clade, referred to as the Eudiscosea (see below, Figs 1, S1,2). The two remaining Flabellinia orders, Himatismenida and Trichosida, never grouped with the Eudiscosea (Table 2) but instead fall in weakly supported relationships outside the Eudiscosea . In addition, two isolates from ATCC ${ }^{\circledR}+$ PRA-29 ${ }^{\text {тм }}$ deposited as Pessonella spp. and a newly described lineage, S. chincoteaguense (Lahr et al., 2015), form a strongly supported clade outside Eudiscosea with weak affinity to the Himatismenida clade (Table 2).

\subsection{Unresolved putative Discosean lineages}




\subsubsection{Himatismenida clade}

The order Himatismenida sensu Smirnov et al. (2011) includes two suborders: Tectiferina (Cochliopodium, Ovalopodium, Gocevia and Paragocevia) and Parvamoebina (Parvamoeba). The taxonomic composition and phylogenetic relationships of Himatismenida is poorly understood (Cavalier-Smith et al., 2004; Kudryavtsev et al., 2005; Kudryavtsev et al., 2011; Page, 1987; Pussard, 1965; Sawyer, 1980; Tekle et al., 2013; Tekle et al., 2015). This is mainly due to limited molecular data, diverse morphologies within the group and rapidly evolving rDNA sequences of its members (e.g. Cochliopodium; Kudryavtsev et al., 2005; Kudryavtsev et al., 2011; Lahr et al., 2011a; Tekle et al., 2008). This high rate of sequence evolution likely contributed to the failure of its members to form a monophyletic clade. Evidence for the current taxonomic composition of Himatismenida is not robust, as it comes from a collection of independent observations from various studies using different data sources (Kudryavtsev, 2012; Kudryavtsev et al., 2014; Smirnov et al., 2011).

Kudryavtsev et al. (2014) transferred Gocevia from Himatismenida into Pellitida based on molecular and morphological evidence. This revision is congruent with our phylogeny where Gocevia never grouped with the rest of the himatismenids (Fig. 1; Table 2). Instead, Gocevia consistently groups within a clade consisting of Acanthamoeba and Stereomyxa with strong support (Fig. 1, Table 2). These taxa are reported to possess a centrosphere, a microtubule-organizing center (MTOC) (Benwitz, 1971; Bowers and Korn, 1968; Pussard, 1977). Similarly, members of Pellitida (not included in our analysis) share this similar feature (Kudryavtsev et al., 2014). Although MTOCs are described from diverse groups of amoebae (Bennett, 1984; Bennett, 1986; Geisen et al., 2014; Grell, 1978) including a himatismenid, Cochliopodium (Kudryavtsev, 2004), the strong support in our molecular analysis for these MTOC containing lineages necessitates a broader re-examination of the homology of MTOC in amoebae to assess its potential use for defining groups. Some of our recent work, based on immunocytochemistry, indicates that the prominence of MTOCs differs among closely related species of amoebae (unpublished data). 
Our analysis provide a strong support for the monophyly of the three himatismenids: Ovalopodium, Cochliopodium and Parvamoeba (Fig. 1, Table 2). While the morphological evidence for sister relationship among these lineages is fairly consistent (Kudryavtsev, 2012; Kudryavtsev et al., 2011), their relationships based on molecular analyses has been poorly resolved (Cole et al., 2010; Kudryavtsev et al., 2005; Kudryavtsev et al., 2011; Tekle et al., 2008). Most SSU-rDNA gene based studies group Ovalopodium and Cochliopodium together (Kudryavtsev et al., 2011), while Cochliopodium and Parvamoeba are recovered as sister groups in the actin phylogeny (Kudryavtsev, 2012). Consistent with the morphological data (ventral thin sheet of hyaloplasm; (Kudryavtsev, 2012) and actin phylogeny, our results support the closer relationships of Cochliopodium and Parvamoeba (Fig. 1). Therefore, relationships showing a close affinity of Ovalopodium and Cochliopodium in SSU-rDNA gene phylogenies is most likely an artifact caused by the highly variable sequences of Cochliopodium and Parvamoeba.

While our analysis demonstrates that the three himatismenids are not members of the 'Discosea' as previously hypothesized, their phylogenetic position within Amoebozoa remains unresolved (Table 2). Given that we were able to place many known problematic long-branched taxa (e.g. Cochliopodium and Mayorella) accurately in the tree, and all AU tests (Table 3) reject their inclusion in 'Discosea', the lack of resolution is less likely due to the effects of long-branch attraction. While future studies with the inclusion of more amoebozoan taxa are needed to resolve this issue, based on the current knowledge of molecular data and morphology, the three himatismenids likely represent an independent lineage within the Amoebozoa.

\subsubsection{Trichosphaerium}

Our analyses were able to resolve the phylogenetic placement of many known problematic amoeboid lineages except Trichosphaerium. This lineage is one of the fastest evolving taxa known in Amoebozoa (Tekle et al., 2008). The currently accepted taxonomic classification of Trichosphaerium is solely based on morphology, placing it within the order Trichosida under Flabellinia/'Discosea' (Smirnov et al., 2011). Similar 
to previous single gene phylogenies, Trichosphaerium cannot be reliably placed within any of the known amoebozoan clades in our analyses (Table 2). Its position varies by analysis and is poorly supported in our trees. Trichosphaerium is also represented by the least amount of data compared to other discosean orders included in our analysis (Table S2). Therefore, placement of Trichosphaerium will require more data.

\subsection{3. $A T C C^{\circledR} P R A-29^{T M}+$ Sapocribrum a novel clade}

Two isolates of ATCC ${ }^{\circledR}$ PRA-29 ${ }^{\mathrm{TM}}$ designated as Pessonella spp. and Sapocribrum chincoteaguense form a robust relationship, but their placement within Amoebozoa is poorly resolved (Table 2). Several molecular phylogenetic studies have shown the sister relationship of these two species (Kudryavtsev, 2013; Lahr et al., 2011a) and in a more recent study both group together with Squamamoeba spp. (Lahr et al., 2015). This novel clade plus Squamamoeba includes lineages with diverse morphology mostly resembling discosean morphotypes, but less so for Sapocribrum, that is largely angular in body shape, but approaches a discoidal form with dorsal surface crenations when not actively locomoting. However, in most molecular analyses they branch as independent lineages with no clear affinity to any of the known discosean lineages (Fig. 1). Lahr et al. (2015) reported a potential synapomorphy of the surface scales observed with TEM, providing evidence for the relationship between Squamamoeba and Sapocribrum. However, little is known about the morphology of the $\mathrm{ATCC}^{\circledR} \mathrm{PRA}-29^{\mathrm{TM}}$ isolate.

The genus Pessonella (Pussard, 1973) characteristically displays a vannellid morphotype with fan-shaped locomotive form, and is currently grouped within the Vannellida (Smirnov and Goodkov, 1999). However, the ATCC ${ }^{\circledR}$ PRA-29 ${ }^{\mathrm{TM}}$ isolate is never observed to group with any of the vannellids (Fig. 1, Table 2). A more detailed reinvestigation of this isolate, beyond the light micrograph images provided in Tekle et al. (2008), might reveal a more accurate identification of this isolate and its evolutionary affinity with Squamamoeba and Sapocribrum. ATCC ${ }^{\circledR}$ PRA-29 ${ }^{T M}$ isolate and Sapocribrum have been consistently observed to group with the three himatismenids, albeit with a very weak support. There is no published report of the fine structure of ATCC ${ }^{\circledR}$ PRA-29 ${ }^{T M}$ isolate, so we cannot make fine structural comparisons between Sapocribrum and ATCC ${ }^{\circledR}$ PRA- 
$29^{\mathrm{TM}}$, especially with respect to surface structures such as the presence or absence of scales. The light microscopic morphology is markedly different for the two amoebae.

\subsection{The Eudiscosea clade}

The Eudiscosea represents an amalgamation of Longamoebia and Flabellinia (Fig. 1). In this revised group, the three orders of Longamoebia are found either nested within a Flabellinia order (e.g. Mayorella in Dactylopodida) or branched as sister taxa to the remaining three orders of Flabellinia (Fig. 1). These new relationships have important evolutionary implications for our understanding of the group as described below.

The placement of Mayorella within Dactylopodida is in agreement with its original classification by F. C. Page (1987). While Mayorella shares important morphological characters with members of Dactylopodida, its placement in previous molecular phylogenetic analyses has proven difficult (e.g. Lahr et al., 2011a). In most molecular analyses based on SSU-rDNA or actin genes that included comprehensive taxon sampling Mayorella's placement varied by analysis and was never shown to group within Dactylopodida (e.g. Kudryavtsev et al., 2014; Lahr et al., 2015; Lahr et al., 2011a; Tekle et al., 2008). In a few published studies with specific taxon sampling, Mayorella grouped with the Dermamoeba, albeit with no or negligible support (Berney et al., 2015; Kudryavtsev et al., 2014). This led Smirnov et al. (2011) to transfer Mayorella into Dermamoebida (Longamoebia) referring to the multilayered cell coat as a potential synapomorphy between them. However, our study refutes both the transfer of Mayorella into Dermamoebida and the monophyly of Longamoebia (Tables 2,3).

A suite of morphological and fine structural features supports the grouping of Mayorella and Vexillifera with the Dactylopodida (e.g. Korotnevella). All three taxa possess a profile characterized by a broad anterior and narrow posterior (Page, 1988). To varying degrees, subpseudopodia emerge from an anterior hyaloplasm, and are initially to some degree conical, but become more finely elongated in Vexillifera, or more dactyloid, partially in Mayorella and especially in Korotnevella. Subpseudopodia are nonfurcated, and tend to migrate toward the posterior of the amoeba, particularly in Vexillifera (Page, 
1988). Subpseudopodia of the latter, however, have a filamentous core (Page, 1979). All bear some form of surface microstructures, such as hexagonal cylindrical in Vexillifera, cylindrical funnel-shaped in Mayorella, and boat-shaped complex scales in Korotnevella. The nucleolus tends to be largely central; although variations occur, such as parietal lobes in Vexillifera (Page, 1983).

Perhaps one of the most stable and consistently recovered major subclades within Eudiscosea (Amoebozoa) in most molecular analyses consists of Dactylopodida and Vannellida (e.g. Fahrni et al., 2003; Peglar et al., 2003; Tekle et al., 2008). Smirnov et al. (2005) formed a taxonomic rank, class Flabellinea, for these two taxa and provided morphological evidence supporting this relationship. Morphological evidence for the newly designated clade Mayorella/Dactylopodida + Vannellida (hereafter referred as clade Flabellinea) based on our analyses include flattened amoebae with polyaxial cytoplasmic flow and members lacking an MTOC (Smirnov et al., 2005). The clade Flabellinea is consistently recovered in all of our analyses with strong support (Fig. 1, Table 2). The two newly characterized vannellids, Clydonella sp. and Unda schaefferi ATCC $^{\circledR} 50810^{\mathrm{TM}}$ are placed within Vannellida as expected (Fig.1). While we are unable to comment on relationships within the Vannellida clade due to low taxonomic sampling, it is interesting to note, however, that the $\mathrm{ATCC}^{\circledR} 50810^{\mathrm{TM}}$ isolate designated as $U$. schaefferi is nested within the Vannella spp. that we have analyzed, making the genus Vannella very likely paraphyletic. The taxonomy and phylogeny of the genus Vannella has been well studied and was recently revised to include Platyamoeba within the genus (Smirnov et al., 2007); with evidence that the characteristic tower-like glycostyles observed in the fine structure of Vannella spp. sensu stricto, are not phylogenetically conservative features. Therefore, in summary, the morphological identity of ATCC ${ }^{\circledR}$ $50810^{\mathrm{TM}}$ isolate needs further reexamination.

A well-supported sister clade to the clade Flabellinea within the Eudiscosea includes the enigmatic genus Vermistella, now with two described species: Vermistella antarctica and Vermistella arctica (Tyml et al., 2015) and two members of the order Thecamoebida ( $S$. stenopodia and T. quadrilineata; Fig. 1). While the close relationship of S. stenopodia 
and $T$. quadrilineata is not new, the placement of $V$. antarctica in previous molecular studies was unstable (Lahr et al., 2011a). Smirnov et al. (2011) placed Vermistella in order Stygamoebida within Flabellinia/'Discosea’ solely based on morphological evidence. Molecular analyses supporting Stygamoeba sister to Vermistella is neither recovered nor could their sister relationship be rejected based on an AU test (Lahr et al., 2011a). Moreover increasing molecular evidence indicates no close phylogenetic relationship between Stygamoeba and Vermistella (e.g. Lahr et al., 2011a; Tyml et al., 2015). The taxonomic validity of Stygamoebida will require a thorough genetic analysis including the phyletic position of Stygamoeba.

Our analyses reveal a novel and unsuspected relationship between Vermistella and Thecamoebida (Fig. 1, Table 2). Morphological and fine structural reevaluation of these lineages indicates some shared features. Both possess a glycocalyx, thicker in Thecamoeba spp., and thinner with closely spaced pyramidal elements in Vermistella (Moran et al., 2007). The thin, electron-dense surface lamina in Vermistella is a feature partially shared with Stenamoeba spp. (Dyková, 2010; Geisen et al., 2014). All three genera have species with a central nucleolus, but the nucleus of Thecamoeba spp. has a thickened, somewhat osmiophilic peripheral lamina on the interior of the nuclear envelope.

The early diverging Eudiscosea clade composed of Acanthamoeba, Stereomyxa and Gocevia - hereafter referred to as clade Centramoebida due to the shared MTOC feature (Fig. 1, Table 2) was recovered with moderately strong support. Gocevia should not be included as a member of Himatismenida, and its affinity to the clade Centramoebida together with Pellita and Endostelium (Pellitida sensu Kudryavtsev et al. 2014) was also observed in a study based on molecular analyses of the SSU-rDNA gene (Kudryavtsev et al., 2014).

The placement of the $\mathrm{ATCC}^{\circledR}$ strain with accession number $50982^{\mathrm{TM}}$ designated as Stereomyxa ramosa has been problematic in a previously published molecular study (e.g. Lahr et al., 2011a) and the taxonomic status of the Stereomyxidae remains poorly 
understood (Benwitz, 1971; Grell, 1966; Lahr et al., 2011a; Smirnov et al., 2011). Stereomyxa ramosa is placed as lobosean incertae sedis in the most recent classification schemes (Adl et al., 2012; Smirnov et al., 2011). Although there is limited morphological data on the $\mathrm{ATCC}^{\circledR} 50982^{\mathrm{TM}}$ isolate, its placement within the clade Centramoebida does not contradict the traditional taxonomic description of Stereomyxidae. Original descriptions report that Stereomyxa possesses an MTOC (Benwitz, 1971) similar to the members of clade Centramoebida. While the presence of an MTOC as a defining feature for this clade is questionable due to its presence in diverse groups of amoebae, the consistent high support of this clade calls for closer reexamination of this feature and other characters that are uniquely shared by this clade.

Although their overall morphology may be quite divergent (varying from flattened, hump-bearing Gocevia to the ramified Stereomyxa), all members of Centramoebida clade share some common morphological details, in addition to the presence of MTOCs. These include a tendency toward branched pseudopodia, more distinctively in fine pseudopodial species (e.g. Acanthamoeba), or in species with broader pseudopodia (Stereomyxa spp. and Gocevia spp.) branching more distally, and may arise from a broader hyaloplasm sheet (Lahr et al. 2011a, Fig. 1y; Rogerson and Patterson, 2002). Fine structural evidence indicates that the plasma membrane of Acanthamoeba, Gocevia, and Stereomyxa is naked and lacks surface elements such as scales or glycostyles. All three have nuclei with a central nucleolus.

\subsection{Taxonomic remark and amendments of the 'Discosea'}

In the last decade and a half we have witnessed a series of minor and major taxonomic revisions within the Amoebozoa as result of molecular phylogenetic studies (CavalierSmith et al., 2004; Kudryavtsev et al., 2014; Lahr et al., 2011a; Smirnov et al., 2005; Smirnov et al., 2011). While this has increased our knowledge of the group, a majority of the revisions are based on single gene (SSU-rDNA) trees with little or no corroboration from other molecular data (e.g. cytoskeletal genes; Tekle et al., 2008). The main challenge of revisions based on SSU-rDNA genes in Amoebozoa is a lack of consistency of inferred phylogenies caused by the observed elevated rate of evolution (Tekle et al., 
2008) and limited signal for deep nodes (Tekle unpublished data). Nevertheless, studies using this gene, only differing in taxonomic sampling, have proposed revisions that resulted in a proliferation of taxonomic names (hypotheses) based on shaky evidence (Smirnov et al., 2005; Smirnov et al., 2011). There are several rDNA-clades that are well corroborated by morphological data but most of the more inclusive taxonomic schemes and proposed relationships within them remain unsupported (Smirnov et al., 2011). Consequences from these revisions based on single genes include inflation of taxonomic names, which creates confusion in the Amoebozoa literature. Therefore, future taxonomic revisions should rely on multiple data sources that reflect phylogenetic relationships. Our study demonstrates that multigene analyses can recover established and novel relationships reliably and consistently with robust support (Table 2).

In this report we propose the revision of 'Discosea' sensu Smirnov et al. (2011) for the following reasons: 1 . Our analyses are taxonomically comprehensive including all nine orders of 'Discosea', 2. Our phylogenies are well resolved and robust to the effects of missing data, choice of methods for analysis and outgroup selection, and 3. Our results are not only congruent with some of the traditionally well-established groups but also some of the novel relationships recovered in this study are corroborated by morphological data. Given more analyses of the Amoebozoa will emerge in the near future, which will allow reconstruction of well-resolved phylogenies, we propose to use a phylogeny based classification scheme similar to that used in Adl et al. (2012). This type of classification system is stable and allows testable phylogenetic frameworks for future revisions.

We provide a stem-modified node-based definition of the taxon name Eudiscosea as follows. The name Eudiscosea is applied to the clade stemming from the most recent common ancestor of the clade Flabellinea (Smirnov et al. 2005, Tekle and Anderson), Thecamoebida (Schaeffer 1926, Tekle and Anderson), Centramoebida (Rogerson \& Patterson 2002, Tekle and Anderson) and all extant organisms that share a more recent common ancestor with the above stated taxa than with the Tubulinea (Smirnov et al. 2005), Variosea (Cavalier-Smith et al., 2004, Smirnov et al., 2008), Mycetozoa (CavalierSmith et al. 2004), Archamoebae (Cavalier-Smith 1983) or Lobosea incertae sedis. This 
definition will allow inclusion of putative discoseans not included in our analyses (e.g. Dermamoeba) or taxa that may fall within this clade upon improved gene and taxon sampling (e.g. former discoseans lineages listed below as Lobosea incertae sedis). Below we list members of Eudiscosea with general shared morphological characters for each group following the scheme of previous publications (Adl et al., 2012; Smirnov et al., 2005; Smirnov et al., 2011). Unresolved discosean lineages such as ATCC ${ }^{\circledR}$ PRA-29 ${ }^{\mathrm{T}}+$ Sapocribrum, Trichosphaerium, and Himatismenida, are listed as Lobosea incertae sedis. Even though these are likely independent lineages within Amoebozoa separate from Eudiscosea (Table 3), our taxonomic sampling and their unresolved position do not justify the creation of a new taxonomic status. Taxa not included in our analyses but where there are morphological or molecular data supporting their potential membership within Eudiscosea are either included as part of (e.g. Pellitida) or incertae sedis (e.g. Dermamoebida) within their respective eudiscosean subclade relatives. In cases where there is not sufficient data supporting a taxon's membership within Eudiscosea, or its monophyly is questioned in a previous study (e.g. Stygamoeba), the taxon is designated as Lobosea incertae sedis.

- Eudiscosea Tekle and Anderson

Clade Eudiscosea includes ((Flabellinea+Thecamoebida)+Centramoebida).

Diagnosis: Flattened naked amoebae, never producing tubular, subcylindrical pseudopodia and never altering the locomotive form; cytoplasmic flow polyaxial or without a pronounced axis; subpseudopodia short or absent, never both pointed and branched.

•• Flabellinea (Smirnov et al. 2005) Tekle and Anderson

Clade Flabellinea includes Dactylopodida (Smirnov et al. 2005) Tekle and Anderson and Vannellida Smirnov et al. 2005.

Diagnosis: Flattened generally fan-shaped, discoid or irregularly triangular, never with pointed subpseudopodia. 
•• Dactylopodida (Smirnov et al. 2005) Tekle and Anderson

Clade Dactylopodida includes Korotnevella, Neoparamoeba, Paramoeba, Pseudoparamoeba, Vexillifera and Mayorella.

Diagnosis: Locomotive form as irregular triangle with broadened base directed forward; wide anterior hyaloplasm; parasomes in Paramoeba and Neoparamoeba; cysts unknown; nonfurcate subpseudopodia emerge, to varying degrees, from an anterior hyaloplasm, and initially to some degree are conical, but may become more finely elongated or more dactyloid, partially as in Mayorella and especially so in Korotnevella, and tend to migrate toward the posterior of the amoeba, particularly in Vexillifera; cortex with extracellular scales, pentagonal or hexagonal glycostyles or a complex fibrous "cuticle".

Note: Squamamoeba is transferred to a clade ATCC ${ }^{\circledR}$ PRA- $29^{\mathrm{TM}}+$ Sapocribrum based on a previous molecular study (Lahr et al. 2015).

••Vannellida Bovee 1979, Smirnov et al. 2005

Clade Vannellida include Clydonella, Lingulamoeba, Pessonella, Protosteliopsis fimicola, Ripella, Vannella, Unda.

Diagnosis: Locomotive form fan-shaped to spatulate; without discrete pseudopodia or subpseudopodia; wide anterior hyaloplasm up to half of the cell; posterior granuloplasm concentrated in a "hump", often raised over the substratum; cell coat is a layer of short glycostyles on top (Clydonella, Lingulamoeba) or pentagonal glycostyles with or without simple filaments (Vannella); one taxon known to be sporocarpic and protosteloid.

Note: the $\operatorname{ATCC}^{\circledR} 50810^{\mathrm{TM}}$ designated as Unda schaefferi is likely a vannellid.

-• Thecamoebida (Schaeffer 1926, Smirnov and Goodkov 1993) Tekle and Anderson

Clade Thecamoebida includes Sappinia, Stenamoeba, Thecamoeba and Vermistella.

Diagnosis: Oblong, flattened cell, and in some cases becoming more elongated to vermiform, surface smooth during locomotion or with dorsal folds and/or ridges; anterior 
hyaloplasm forms antero-lateral crescent and never occupies half or more of the body length; never produces discrete pseudopodia or subpseudopodia; cell coat electron dense and relatively thin to thickened, amorphous or with extra structures including closely spaced electron dense subunits.

Note: Vermistella previously placed under order Stygamoebida (Smirnov et al. 2011) is transferred to clade Thecamoebida.

- Centramoebida (Rogerson \& Patterson 2002, Cavalier-Smith et al. 2004) Tekle and Anderson

Clade Centramoebida includes Acanthamoeba, Balamuthia, Protacanthamoeba, Stereomyxa and members of Pellitida sensu Kudryavtsev et al. (2014): Pellitidae, Goceviidae and Endostelium.

Diagnosis: Flattened with prominent subpseudopodia, flexible and tapering to a fine tip and sometimes furcated near their base (acanthopodia), or with more distal, broader lobose furcated subpseudopodia, sometimes emerging from a hyaloplasm sheet; without adhesive uroid; trilaminate cytoplasmic microtubular organizing center (MTOC); flattened sheet without subpseudopodia; at least one species sporocarpic and protosteloid; generally plasma membrane naked without surface scales or styles, although surface coats of varying organization occur in some taxa.

Note: while there is sufficient molecular and morphological evidence for the inclusion of Pellitida in clade Centramoebida, the membership of Dermamoebida among members of the newly defined clade Centramoebida will require further study. Dermamoebida is designated here as clade Centramoebida incertae sedis.

\section{Lobosea Incertae sedis}

- Himatismenida Page 1987

Clade Himatismenida includes ((Cochliopodium+Parvamoeba $)+$ Ovalopodium $)$.

Diagnosis: Dorsal surface covered with a flexible cuticle or tectum, sometimes scale- 
bearing, without defined aperture; ventral surface naked.

- Trichosphaerium Schneider 1878

Diagnosis: Cell enveloped with flexible membranous test (smooth form) or membranous test of fibrils and matrix bearing spicules (spicule-bearing form); both types of envelopes are separated from the plasma membrane; the amoeba protrudes through this envelope with temporary openings, producing finger-shaped dactylopodia.

- $\quad$ ATCC ${ }^{\circledR}$ PRA- $29^{\mathrm{TM}}+$ Sapocribrum clade

Clade ATCC ${ }^{\circledR}$ PRA-29 ${ }^{\mathrm{TM}}+$ Sapocribrum includes ATCC ${ }^{\circledR}$ PRA-29 ${ }^{\mathrm{TM}}$, Sapocribrum and Squamamoeba.

Diagnosis: Morphologically diverse, mostly resembling discosean morphotypes, apparently closely adherent to substratum, but less so for Sapocribrum that tends to vary from reniform to discoidal with dorsal surface crenations and extending a slender, anterior furcate pseudopodium during locomotion. Surface scales, when present, oval, thin, flattened or margins slightly bent distally.

- Stygamoebida Smirnov et al. 2011

Diagnosis: Flattened, elongate amoebae resembling tooth-pick or splinters, temporarily acquiring forked or branched form; extended area of anterior hyaloplasm; presently, based on our revised analyses, includes only Stygamoeba (Sawyer, 1975; Smirnov, 1995).

Note: Stygamoeba is the sole member of a lobosean incertae sedis, Stygamoebida.

\section{Acknowledgments}

This work is supported by the National Science Foundation RIA Grant (1409587) to YIT. We would like to thank Jessica Grant for assistance with data analysis; the ATCC team including Kathleen C. Glaser and Stephen King for assistance in data collection. We are thankful to the two anonymous reviewers for their constructive comments. This is 
Lamont-Doherty Earth Observatory Contribution Number 0000.

\section{Figure Legend}

Fig. 1: Phylogenomic reconstruction of the 'Discosea' based on the concatenated fulldataset (199347 aa, 550 genes) including 40 amoebozoan taxa reconstructed using RAxML. Newly characterized taxa from this study are shown in bold. Branches labels are RAxML bootstrap support. All branches are drawn to scale expect those indicated branched that were trimmed to half-length for display purposes. 


\section{References}

Adl, S.M., Simpson, A.G., Lane, C.E., Lukes, J., Bass, D., Bowser, S.S., Brown, M.W., Burki, F., Dunthorn, M., Hampl, V., Heiss, A., Hoppenrath, M., Lara, E., Le Gall, L., Lynn, D.H., McManus, H., Mitchell, E.A., Mozley-Stanridge, S.E., Parfrey, L.W., Pawlowski, J., Rueckert, S., Shadwick, R.S., Schoch, C.L., Smirnov, A., Spiegel, F.W., 2012. The revised classification of eukaryotes. The Journal of eukaryotic microbiology 59, 429-493.

Amaral Zettler, L.A., Nerad, T.A., O'Kelly, C.J., Peglar, M.T., Gillevet, P.M., Silberman, J.D., Sogin, M.L., 2000. A molecular reassessment of the leptomyxid amoebae. Protist 151, 275-282.

Bennett, W., 1984. Fine structure of the trophic stage of Endostelium Olive, Bennett \& Deasey, (Eumycetozoea, Protosteliia). Protistologica 22, 205-212.

Bennett, W.E., 1986. An ultrastractural study of the trophozoiteand cyst stages of Protostelium pyriformis Olive \& Stoianovitch, 1969 (Eumycetozoea, Protosteliia). . J Protozool 33, 405-411.

Benwitz, G., Grell, K. , 1971. Ultrastruktur mariner Amöben III. Stereomyxa angulosa. Arch Protistenkd 113, 68-79.

Berger, S.A., Krompass, D., Stamatakis, A., 2011. Performance, accuracy, and Web server for evolutionary placement of short sequence reads under maximum likelihood. Systematic Biology 60, 291-302.

Berney, C., Geisen, S., Van Wichelen, J., Nitsche, F., Vanormelingen, P., Bonkowski, M., Bass, D., 2015. Expansion of the 'Reticulosphere': Diversity of Novel Branching and Network-forming Amoebae Helps to Define Variosea (Amoebozoa). Protist 166, 271-295.

Bolger, A.M., Lohse, M., Usadel, B., 2014. Trimmomatic: a flexible trimmer for Illumina sequence data. Bioinformatics 30, 2114-2120.

Bowers, B., Korn, E.D., 1968. The fine structure of Acanthamoeba castellanii. I. The trophozoite. J Cell Biol 39, 95-111.

Cavalier-Smith, T., Chao, E.E.Y., Oates, B., 2004. Molecular phylogeny of Amoebozoa and the evolutionary significance of the unikont Phalansterium. European journal of protistology 40, 21-48.

Cavalier-Smith, T., Fiore-Donno, A.M., Chao, E., Kudryavtsev, A., Berney, C., Snell, E.A., Lewis, R., 2015. Multigene phylogeny resolves deep branching of Amoebozoa. Mol Phylogenet Evol. 83, 293-304.

Cole, J., Anderson, O.R., Tekle, Y.I., Grant, J., Kat, L.A., Nerad, T., 2010. A description of a new "Amoebozoan" isolated from the American lobster, Homarus americanus. The Journal of eukaryotic microbiology 57, 40-47.

Darriba, D., Taboada, G.L., Doallo, R., Posada, D., 2011. ProtTest 3: fast selection of best-fit models of protein evolution. Bioinformatics 27, 1164-1165.

Dyková, I., Kostka, M., Pecková, H. , 2010. Two new species of the genus Stenamoeba Smirnov, Nassonova, Chao et Cavalier-Smith, 2007. Acta Protozool. 49, 245-251.

Eichinger, L., Pachebat, J.A., Glockner, G., Rajandream, M.A., Sucgang, R., Berriman, M., Song, J., Olsen, R., Szafranski, K., Xu, Q., Tunggal, B., Kummerfeld, S., Madera, M., Konfortov, B.A., Rivero, F., Bankier, A.T., Lehmann, R., Hamlin, N., 
Davies, R., Gaudet, P., Fey, P., Pilcher, K., Chen, G., Saunders, D., Sodergren, E., Davis, P., Kerhornou, A., Nie, X., Hall, N., Anjard, C., Hemphill, L., Bason, N., Farbrother, P., Desany, B., Just, E., Morio, T., Rost, R., Churcher, C., Cooper, J., Haydock, S., van Driessche, N., Cronin, A., Goodhead, I., Muzny, D., Mourier, T., Pain, A., Lu, M., Harper, D., Lindsay, R., Hauser, H., James, K., Quiles, M., Madan Babu, M., Saito, T., Buchrieser, C., Wardroper, A., Felder, M., Thangavelu, M., Johnson, D., Knights, A., Loulseged, H., Mungall, K., Oliver, K., Price, C., Quail, M.A., Urushihara, H., Hernandez, J., Rabbinowitsch, E., Steffen, D., Sanders, M., Ma, J., Kohara, Y., Sharp, S., Simmonds, M., Spiegler, S., Tivey, A., Sugano, S., White, B., Walker, D., Woodward, J., Winckler, T., Tanaka, Y., Shaulsky, G., Schleicher, M., Weinstock, G., Rosenthal, A., Cox, E.C., Chisholm, R.L., Gibbs, R., Loomis, W.F., Platzer, M., Kay, R.R., Williams, J., Dear, P.H., Noegel, A.A., Barrell, B., Kuspa, A., 2005. The genome of the social amoeba Dictyostelium discoideum. Nature 435, 43-57.

Fahrni, J.F., Bolivar, I., Berney, C., Nassonova, E., Smirnov, A., Pawlowski, J., 2003. Phylogeny of lobose amoebae based on actin and small-subunit ribosomal RNA genes. Molecular biology and evolution 20, 1881-1886.

Felsenstein, J., 1978. Cases in which parsimony or compatibility methods will be positively misleading. Systematic Zoology 27, 401-410.

Geisen, S., Weinert, J., Kudryavtsev, A., Glotova, A., Bonkowski, M., Smirnov, A., 2014. Two new species of the genus Stenamoeba (Discosea, Longamoebia): cytoplasmic MTOC is present in one more amoebae lineage. European journal of protistology 50, 153-165.

Grant, J.R., Katz, L.A., 2014. Building a phylogenomic pipeline for the eukaryotic tree of life - addressing deep phylogenies with genome-scale data. PLoS currents 6.

Grell, K., Benwitz, G., 1978. Ultrastruktur mariner Amöben IV.Corallomyxa chattoni. . Arch Protistenkd 120, 287-300.

Grell, K.G., 1966. Amöben der Familie Stereomyxidae. Arch. Protistenk. 109, 147-154.

Kudryavtsev, A., 2004. Description of Cochliopodium spiniferum sp. n., with notes on the species identification within the genus Cochliopodium. Acta Protozool. 43, 345349.

Kudryavtsev, A., 2012. Microscopic evidence for inclusion of Parvamoeba Rogerson, 1993 into the order Himatismenida (Amoebozoa). European journal of protistology $48,85-88$.

Kudryavtsev, A., Bernhard, D., Schlegel, M., Chao, E.E.Y., Cavalier-Smith, T., 2005. $18 \mathrm{~S}$ ribosomal RNA gene sequences of Cochliopodium (Himatismenida) and the phylogeny of Amoebozoa. Protist 156, 215-224.

Kudryavtsev, A., Brown, M.W., Tice, A., Spiegel, F.W., Pawlowski, J., Anderson, O.R., 2014. A revision of the order Pellitida Smirnov et al., 2011 (Amoebozoa, Discosea) based on ultrastructural and molecular evidence, with description of Endostelium crystalliferum n. sp. Protist 165, 208-229.

Kudryavtsev, A., Pawlowski, J., 2013. Squamamoeba japonican g. n. sp. (Amoebozoa): a deep-sea amoeba from the Sea of Japan with a novel cell coat structure. Protist 164, $13-23$. 
Kudryavtsev, A., Wylezich, C., Pawlowski, J., 2011. Ovalopodium desertum n. sp. and the phylogenetic relationships of Cochliopodiidae (Amoebozoa). Protist 162, 571589.

Lahr, D.J., Grant, J., Molestina, R., Katz, L.A., Anderson, O.R., 2015. Sapocribrum chincoteaguense n. gen. n. sp.: A Small, Scale-bearing Amoebozoan with Flabellinid Affinities. The Journal of eukaryotic microbiology 62, 444-453.

Lahr, D.J., Grant, J., Nguyen, T., Lin, J.H., Katz, L.A., 2011a. Comprehensive phylogenetic reconstruction of amoebozoa based on concatenated analyses of SSUrDNA and actin genes. PloS one 6, e22780.

Lahr, D.J., Grant, J.R., Katz, L.A., 2013. Multigene phylogenetic reconstruction of the Tubulinea (Amoebozoa) corroborates four of the six major lineages, while additionally revealing that shell composition does not predict phylogeny in the Arcellinida. Protist 164, 323-339.

Lahr, D.J., Nguyen, T.B., Barbero, E., Katz, L.A., 2011b. Evolution of the actin gene family in testate lobose amoebae (Arcellinida) is characterized by two distinct clades of paralogs and recent independent expansions. Molecular biology and evolution 28, 223-236.

Lahr, D.J.G., Kubik, G.M., Gant, A.L., Grant, J., Anderson, O.R., Katz, L.A., 2012. Morphological description of Telaepolella tubasferens n. g., n. sp., isolate ATCC(C 50593TM, a filose amoeba in the Gracilipodida, Amoebozoa. Acta Protozool 51, 305-318.

Lartillot, N., Brinkmann, H., Philippe, H., 2007. Suppression of long-branch attraction artefacts in the animal phylogeny using a site-heterogeneous model. BMC evolutionary biology 7 Suppl 1, S4.

Lee, J.J., Hutner, S.H., Bovee, E.C., 1985. An illustrated guide to the protozoa. Society of Protozoologists, Lawrence, KS.

Maddison, D.R., Maddison, W.P., 2005. MacClade version 4.08: an analysis of phylogeny and character evolution. Sinauer Associates, Sunderland, MA.

Miller, M.A., Pfeiffer, W., and Schwartz, T., 2010. Creating the CIPRES Science Gateway for inference of large phylogenetic trees. in Proceedings of the Gateway Computing Environments Workshop (GCE), pp 1 - 8.

Moran, D.M., Anderson, O.R., Dennett, M.R., Caron, D.A., Gast, R.J., 2007. A description of seven Antarctic marine gymnamoebae including a new subspecies, two new species and a new genus: Neoparamoeba aestuarina antarctica n. subsp., Platyamoeba oblongata n. sp., Platyamoeba contorta n. sp. and Vermistella antarctica n. gen. n. sp. The Journal of eukaryotic microbiology 54, 169-183.

Nikolaev, S.I., Berney, C., Petrov, N.B., Mylnikov, A.P., Fahrni, J.F., Pawlowski, J., 2006. Phylogenetic position of Multicilia marina and the evolution of Amoebozoa. International Journal of Systematic and Evolutionary Microbiology 56, 1449-1458.

Page, F.C., 1979. Vexillifera armata new species gymnamoebia paramoebidae an estuarine amoeba with distinctive surface structures and trichocyst like bodies. Protistologica 15, 111-122.

Page, F.C., 1983. Marine Gymnamoebae. Cambridge: Institute of Terrestrial Ecology. Page, F.C., 1987. The classification of 'naked' amoebae (Phylum Rhizopoda). Arch. Protistenkd. 133, 199-217. 
Page, F.C., 1988. A New Key to Freshwater and Soil Gymnamoebae. Cumbria: Freshwater Biological Association.

Peglar, M.T., Amaral Zettler, L.A., Anderson, O.R., Nerad, T.A., Gillevet, P.M., Mullen, T.E., Frasca Jr., S., Silberman, J.D., O'Kelly, C.J., Sogin, M.L., 2003. Two new small-subunit ribosomal RNA gene lineages within the Subclass Gymnamoebia. J. Eukaryot. Microbiol. 50, 224-232.

Pisani, D., 2004. Identifying and removing fast-evolving sites using compatibility analysis: an example from the Arthropoda. Syst. Biol. 53, 978-989.

Pussard, M., 1965. Cuticule et caryocinèse de Gocevia fonbrunei, n. sp. (Cochliopodiidae, Testacealobosa). Arch Zool Exp Gen 105, 101-107.

Pussard, M., Senaud, J., Pons, R., 1977. Observations ultrastructurales sur Gocevia fonbrunei Pussard 1965 (Protozoa, Rhizopodea). Protistologica 13, 265-285.

Rogerson, A., Patterson, D.J., 2002. The Naked Ramicristate Amoebae (Gymnamoebae). In: Lee, J.J., Leedale, G.F., Bradbury, P. (Eds.), An Illustrated Guide to the Protozoa, 2nd ed. Society of Protozoologists,Lawrence, Kansas, pp. 1023-1053.

Ronquist, F., Huelsenbeck, J.P., 2003. MrBayes 3: Bayesian phylogenetic inference under mixed models. Bioinformatics 19, 1572-1574.

Sawyer, T.K., 1975. Marine amoebae from surface waters of chincoteague bay, Virginia: one new genus and eleven new species within the families Thecamoebidae and Hyalodiscidae. Trans Am Microsc Soc 94, 305-323.

Sawyer, T.K., 1980. Marine amebae from clean and stressed bottom sediments of the Atlantic Ocean and Gulf of Mexico. J Protozool 27, 13-32.

Schaeffer, A.A., 1926. Taxonomy of the Amebas. Papers Dept Mar Biol Carnegie Inst Wash 24, 3-112.

Shimodaira, H., 2002. An approximately unbiased test of phylogenetic tree selection. Syst. Biol. 51, 492-508.

Shimodaira, H., Hasegawa, M., 2001. CONSEL: for assessing the confidence of phylogenetic tree selection. Bioinformatics 17, 1246-1247.

Smirnov, A., Nassonova, E., Berney, C., Fahrni, J., Bolivar, I., Pawlowski, J., 2005. Molecular phylogeny and classification of the lobose amoebae. Protist 156, 129-142.

Smirnov, A.V., 1995. Stygamoeba regulata $\mathrm{n}$. sp. (Rhizopoda) - a marine amoeba with an unusual combination of light-microscopical and ultrastructural features. Arch. Protistenkd. 146, 299-307.

Smirnov, A.V., Chao, E., Nassonova, E.S., Cavalier-Smith, T., 2011. A revised classification of naked lobose amoebae (Amoebozoa: lobosa). Protist 162, 545-570.

Smirnov, A.V., Goodkov, A.V., 1999. An Illustrated list of basic morphotypes of Gymnamoebia (Rhizopoda, Lobosea). Protistology 1, 20-29.

Smirnov, A.V., Nassonova, E.S., Chao, E., Cavalier-Smith, T., 2007. Phylogeny, evolution, and taxonomy of vannellid amoebae. Protist 158, 295-324.

Stamatakis, A., Hoover, P., Rougemont, J., 2008. A Rapid Bootstrap Algorithm for the RAxML Web-Servers. Syst. Biol. 57, 758-771.

Stamatakis, A., Ludwig, T., Meier, H., 2005. RAxML-III: a fast program for maximum likelihood-based inference of large phylogenetic trees. Bioinformatics 21, 456-463.

Stanley, S.L., Jr., 2005. The Entamoeba histolytica genome: something old, something new, something borrowed and sex too? Trends in parasitology 21, 451-453. 
Tekle, Y.I., Anderson, O.R., Lecky, A.F., Kelly, S.D., 2013. A New Freshwater Amoeba: Cochliopodium pentatrifurcatum n. sp. (Amoebozoa, Amorphea). J Eukaryot Microbiol. 60, 342-349.

Tekle, Y.I., Gorfu, L.A., Anderson, O.R., 2015. Cochliopodium arabianum n. sp. (Amorphea, Amoebozoa). J Eukaryot Microbiol. 62(5), 623-8.

Tekle, Y.I., Grant, J., Anderson, O.R., Nerad, T.A., Cole, J.C., Patterson, D.J., Katz, L.A., 2008. Phylogenetic placement of diverse amoebae inferred from multigene analyses and assessment of clade stability within 'Amoebozoa' upon removal of varying rate classes of SSU-rDNA. Mol Phylogenet Evol. 47, 339-352.

Tyml, T., Kostka, M., Ditrich, O., Dykova, I., 2015. Vermistella arctica n. sp. Nominates the Genus Vermistella as a Candidate for Taxon with Bipolar Distribution. J Eukaryot Microbiol. doi: 10.1111/jeu.12270. 
Table 1. Information on the RNA-Seq data from six Amoebozoa characterized for this study.

\begin{tabular}{lllll}
\hline Taxon & Source & \# of reads & \# of contigs & Bioproject \# \\
\hline Clydonella sp. & ATCC 50884 & $17.2 \mathrm{M}$ & 5386 & xxxxxxxxxx \\
Gocevia fonbrunei & ATCC 50196 & $5.3 \mathrm{M}$ & 4685 & xxxxxxxxx \\
Unda schaefferi & ATCC 50810 & $6.7 \mathrm{M}$ & 3877 & xxxxxxxxx \\
Thecamoeba quadrilineata & ATCC PRA-259 & $13.2 \mathrm{M}$ & 7298 & xxxxxxxxx \\
Parvamoeba monoura & ATCC PRA-35 & $15.2 \mathrm{M}$ & 5500 & xxxxxxxxx \\
Vermistella antarctica & ATCC PRA-216 & $35 \mathrm{M}$ & 5967 & xxxxxxxxx \\
\hline
\end{tabular}


Table 2. Support value (BS) for some of the proposed taxonomic hypotheses within 'Discosea', Amoebozoa and placement of enigmatic taxa based on analyses with different degrees of missing (masking) data matrices including full (550 genes with 199347 aa), 25\%-mask (496 genes with 149123 aa) and 50\%-mask (400 genes with 97059 aa).

Taxonomic hypothesis and classification follows Smirnov et al. 2011 unless indicated.

\begin{tabular}{|c|c|c|c|}
\hline Taxonomic hypothesis & Full & $25 \%$-mask & 50\%-mask \\
\hline Amoebozoa & 100 & 100 & 100 \\
\hline Conosa & $\mathrm{nm}$ & $\mathrm{nm}$ & $\mathrm{nm}$ \\
\hline Mycetozoa & 100 & 100 & 100 \\
\hline Varipodia & 100 & 100 & 100 \\
\hline Mycetozoa+Varipodia & 54 & 88 & $\mathrm{~nm}$ \\
\hline Tubulinea & 62 & 93 & $\mathrm{~nm}$ \\
\hline 'Discosea' & $\mathrm{nm}$ & $\mathrm{nm}$ & $\mathrm{nm}$ \\
\hline Flabellinia & $\mathrm{nm}$ & $\mathrm{nm}$ & $\mathrm{nm}$ \\
\hline Longamoebia & $\mathrm{nm}$ & $\mathrm{nm}$ & $\mathrm{nm}$ \\
\hline Vannellida+*Dactylopodida & 100 & 100 & 94 \\
\hline Mayorella in Dactylopodida & 100 & 100 & 100 \\
\hline Vermistella in Thecamoebida & 100 & 100 & 99 \\
\hline Gocevia $(\mathbf{G})+($ Stereomyxa $(\mathbf{S})+$ Centramoebida $(\mathbf{C}))$ & 100 & 100 & 100 \\
\hline **Eudiscosea & 87 & 87 & 95 \\
\hline Himatismenida & 98 & 100 & 99 \\
\hline Pessonella+Sapocribrum & 100 & 100 & 100 \\
\hline$($ Pessonella + Sapocribrum $)+$ Himatismenida & 41 & 42 & 53 \\
\hline Trichosphaerium in Eu-/'Discosea' & - & - & - \\
\hline Himatismenida in Eu-/‘Discosea' & - & - & - \\
\hline Pessonella+Sapocribrum in Eu-/'Discosea' & - & - & - \\
\hline
\end{tabular}

NOTES: *Dactylopodida $=$ Dactylopodida + Mayorella $; * *$ Eudiscosea - is newly defined clade consisting of $((($ Vannellida+(Dactylopodida+Mayorella $))+($ Thecamoebida + Vermistella $))+(\mathrm{G}+(\mathrm{S}+\mathrm{C})))$ 
Table 3. Results of the Approximately Unbiased (AU) test reveal none of the alternative traditional taxonomic hypotheses are supported.

\begin{tabular}{|c|c|c|c|}
\hline Hypothesis & au & wkh & wsh \\
\hline Eudiscosea & 0.701 & 0.624 & 0.981 \\
\hline 'Discosea' & $4 \mathrm{e}-05$ & 0 & 0 \\
\hline Eudiscosea+Trichosphaerium $s p$. & $7 e-05$ & 0 & 0 \\
\hline Eudiscosea+Himatismenida & $1 e-32$ & 0 & 0 \\
\hline Eudiscosea+(Pessonella+Sapocribrum) & $6 e-07$ & 0 & 0 \\
\hline $\begin{array}{l}\text { Eudiscosea }+ \\
\text { (Himatismenida }+ \text { Pessonella }+ \text { Sapocribrum) }\end{array}$ & 0.001 & 0 & 0 \\
\hline $\begin{array}{l}\text { (Eudiscosea+Himatismenida)+ } \\
\text { Pessonella }+ \text { Sapocribrum }\end{array}$ & $3 e-06$ & 0 & 0 \\
\hline $\begin{array}{l}(\text { Eudiscosea }+ \text { Pessonella }+ \text { Sapocribrum })+ \\
\text { Himatismenida }\end{array}$ & $2 \mathrm{e}-59$ & 0 & 0 \\
\hline
\end{tabular}

Notes: The seven alterantive tested for monophyly are presented below the most likely tree generated in this study. au - Approximatelly unbiased test; wkh - weighted Kishino- Hasegawa test; wsh - weighted Shimodaira-Hasegawa test. 


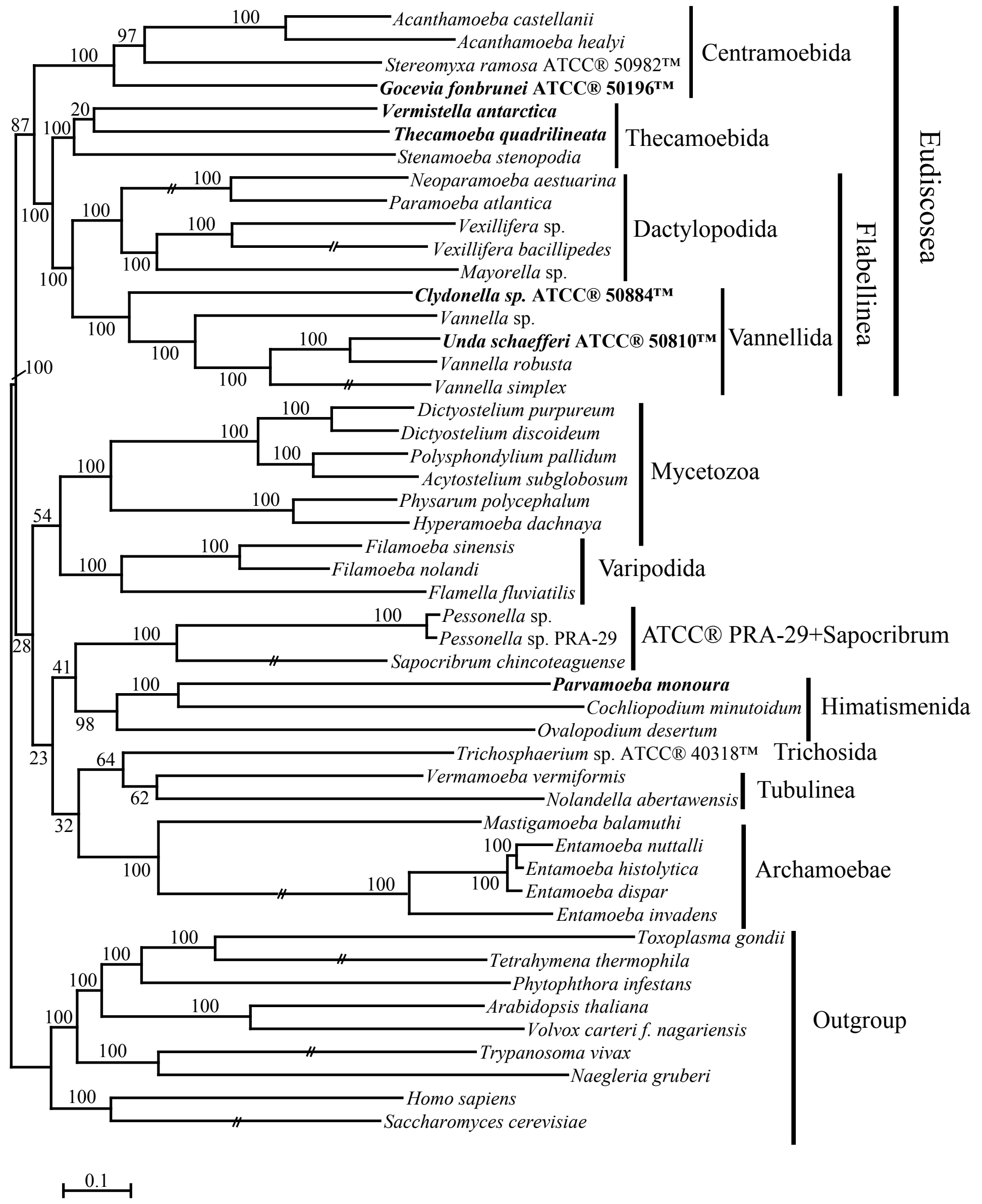




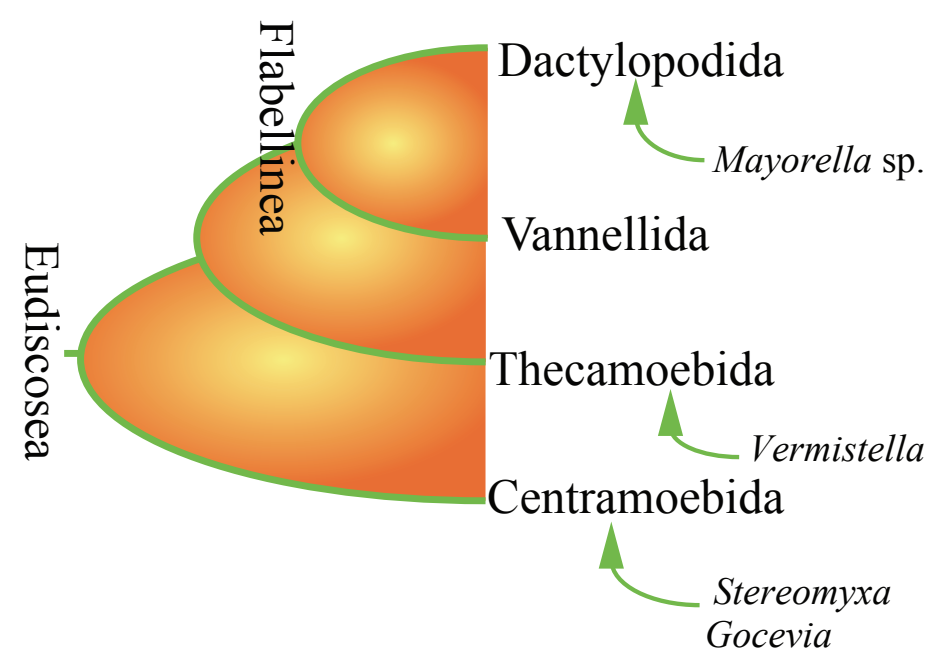

